\title{
Spectral and photometric evolution of simple stellar populations at various metallicities
}

\author{
J. Schulz, U. Fritze - v. Alvensleben, C. S. Möller, and K. J. Fricke \\ Universitätssternwarte Göttingen, Geismarlandstr. 11, 37083 Göttingen, Germany ${ }^{\star}$
}

Received 24 July 2001 / Accepted 25 April 2002

\begin{abstract}
A new set of evolutionary synthesis spectra are presented for Simple Stellar Populations (SSPs) covering ranges in metallicity from $0.02 \leq Z / Z_{\odot} \leq 2.5$ and ages from $4 \times 10^{6} \mathrm{yr} \leq t \leq 16 \mathrm{Gyr}$. They are based on the most recent isochrones from the Padova group that extend earlier models by the inclusion of the thermal pulsing AGB phase for stars in the mass range $2 M_{\odot} \leq m \leq 7 M_{\odot}$ in accordance with the fuel consumption theorem. We show that with respect to earlier models, inclusion of the TP-AGB phase leads to significant changes in the $(V-I)$ and $(V-K)$ colors of SSPs in the age range from $10^{8}$ to $\gtrsim 10^{9} \mathrm{yr}$. Using model atmosphere spectra from Lejeune et al. $(1997,1998)$, we calculate the spectral evolution of single burst populations of various metallicities covering the wavelength range from $90 \AA$ through $160 \mu \mathrm{m}$. Isochrone spectra are convolved with filter response functions to describe the time evolution of luminosities and colors in Johnson, Thuan \& Gunn, Koo, HST, Washington and Strömgren filters. The models and their results are not only intended for use in the interpretation of star clusters but also for combination with any kind of dynamical galaxy formation and/or evolution model that contains a star formation criterion. Moreover, the evolution of these single burst single metallicity stellar populations is readily folded with any kind of star formation - and eventually chemical enrichment - history to describe the evolutionary spectral synthesis of composite stellar populations like galaxies of any type with continuous or discontinuous star formation. For these latter purposes we also present the time evolution of ejection rates for gas and metals for two different Initial Mass Functions (IMFs) as well as cosmological and evolutionary corrections for all the filters as a function of redshift for $0 \leq z \leq 5$ and two different cosmologies. Extensive data files are provided in the electronic version, at CDS, and at our above www-address.
\end{abstract}

Key words. globular clusters: general - open clusters and associations: general - galaxies: star clusters - galaxies: evolution

\section{Introduction}

SSPs are single burst single metallicity stellar populations like star clusters with all their stars having the same age and the same metallicity. Evolutionary synthesis models describe the evolution of these SSPs in terms of photometry and spectra. They are of interest for several reasons. 1) They allow to test a set of stellar evolutionary tracks against observations of star clusters from young open clusters to old Globular Clusters (GCs). 2) They are used for the interpretation of star clusters formed or forming in strong starbursts like those accompanying galaxy interactions or mergers. Being formed from the pre-enriched ISM of the merging galaxies, these young star clusters may cover a wide range in metallicity. 3) Evolutionary models for individual stellar generations can directly be folded - in terms of luminosity or spectral evolution - with any Star Formation History (SFH) to describe the composite stellar population of a galaxy. Or, the other way round, a complete library of spectra for SSPs of various ages and metallicities provides a theoretical basis for population

\footnotetext{
Send offprint requests to: U. Fritze - v. Alvensleben,

e-mail: ufritze@uni-sw.gwdg.de

^ http://www.uni-sw.gwdg.de/ galev/
}

synthesis models. Population Synthesis models search for a particular linear combination of input spectra that gives best agreement with the observed spectrum of a galaxy with unknown SFH. 4) Evolutionary synthesis results for SSPs can be used in any kind of dynamical galaxy formation and/or evolution code that involves a star formation criterion. An example is presented in Contardo et al. (1999), where - in the context of a cosmological structure formation scenario - an individual galaxy is followed from the onset of Star Formation (SF) in a couple of subgalactic fragments to a state where it resembles the Milky Way in many respects. Spatially resolved observable quantities are obtained from a superposition of SSP spectrophotometric evolutionary model results.

A basic difficulty for single burst models as opposed to models for galaxies - which always have extended and continuous SFHs - is the discreteness of the stellar mass spectrum for which evolutionary tracks have been calculated. Lifetime differences between stars for which stellar evolutionary tracks are available are of the order of $\geq 5 \times 10^{8} \mathrm{yr}$, i.e. much longer than the age spread among the stars in SSP models with burst durations of $\leq 10^{5} \mathrm{yr}$ and also much longer than a typical timestep in the evolutionary synthesis model. In late evolutionary phases, this causes large numbers of stars to collectively enter and leave 
distinct phases of evolution with the effect of significant oscillations in colors and magnitudes. These oscillations in the photometric properties have been cured in different ways. E.g., they can be smoothed a posteriori. This has the advantage that the intrinsic uncertainty of the method remains clearly visible. A smooth spectral evolution, however, is difficult to obtain. A second possibility to start from a discrete set of stellar tracks and obtain a reasonably smooth photometric evolution is the use of a Monte Carlo method as described e.g. by Kurth et al. (1999). This method works very well for a photometric description but requires a huge number of Monte Carlo stars and timesteps to yield a smooth spectral evolution. The third possibility is the use of isochrones obtained on the basis of a grid of stellar evolutionary tracks by interpolating between equivalent evolutionary stages. Once the definition of equivalent evolutionary stages is taken for granted, this method provides a smooth evolution of SSPs, both in terms of photometry and spectra.

Here we present a new set of evolutionary synthesis models for the photometric and spectral evolution of SSPs of various metallicities $0.02 \leq Z / Z_{\odot} \leq 2.5$ over a Hubble time, based on the latest Padova isochrones (cf. Sect. 2.2). In this sense, our models are complementary to Leitherer et al.'s (1999) Starburst 99 models which provide a very detailed description of the earliest evolutionary phases $(\leq 1 \mathrm{Gyr})$ of instantaneous burst populations (=SSPs). In Sect. 2, we present our models and the input physics we use. Section 3 gives the spectral evolution of SSPs for 5 different metallicities from $10^{6}$ yrs to $10^{10} \mathrm{yr}$ and a discussion of the effects of metallicity on the time evolution of the spectra. In Sect. 4, we present the evolution of photometric quantities for a number of widely used filter systems. In Sect. 5, we compare our results to earlier models and GC data. In Sect. 6, we present cosmological and evolutionary corrections for our SSP models in 2 different cosmologies in a way that can immediately be used by observers or included into dynamical galaxy formation and evolutionary codes.

Only a few selected figures are presented here, the full tables for the time evolution of spectra, luminosities, colors, ejection rates, etc. of SSPs of all metallicities as well as the corresponding evolutionary and cosmological corrections as a function of redshift can be found at our webpage (http: //www . uni-sw.gwdg.de/ galev/).

Our isochrone synthesis code is able to describe the evolution of stellar populations in galaxies with extended SFHs, as well. In this paper, however, we only present SSP model results.

\section{Models and input physics}

\subsection{Input physics: IMF}

Results in this paper are presented for two different forms of the stellar IMF. While a Salpeter IMF is parametrised as usual $\Phi(m) \sim m^{-2.35}$ over the range of stellar masses $m$ from $m_{\mathrm{l}}=0.15 M_{\odot}$ to $m_{\mathrm{u}} \approx 85 M_{\odot}$, a Scalo IMF is used in the form $\Phi(m) \sim m^{-x}$ with $x=-1.25$ for $m \leq 1 M_{\odot}, x=-2.35$ for $1 M_{\odot}<m \leq 2 M_{\odot}$, and $x=-3.00$ for $m>2 M_{\odot}$. Normalization in both cases is $\int_{m}^{m_{\mathrm{u}}} \Phi(m) \cdot m \cdot \mathrm{d} m=1$. This implies that - as compared to a Salpeter IMF - the Scalo IMF has more stars in the mass range $0.5 M_{\odot} \leq m \leq 6.5 M_{\odot}$ and less stars below $0.5 M_{\odot}$ and above $6.5 M_{\odot}$.

\subsection{Input physics: Padova isochrones with TP-AGB}

The results from our evolutionary synthesis model are based on isochrones from November, 18th, 1999 of the Padova group (for a description and discussion of parameters for these isochrones with moderate core overshooting see Bertelli et al. 1994 and Girardi et al. 2000). Comparison of these isochrones with CMDs of resolved star clusters in the Milky Way and LMC were e.g. presented by Chiosi et al. (1992) and Vallenari et al. (1994). We used the following data from their files: mass, $\log \left(T_{\mathrm{E}}\right), M_{\mathrm{Bol}}$, and $M_{V}$ in their time evolution. We do not use the colors and other luminosities also contained in their files, because we obtain them from the spectra we calculate.

For the use with single short burst stellar populations, isochrones have a basic advantage over the stellar evolutionary tracks they are based on. Because tracks are only available for typically 50 (or less) stellar masses, drastic changes in the luminosity evolution of an SSP occur when all stars of a certain mass collectively climb on the red giant branch or die. Between reasonably defined equivalent evolutionary stages of two stellar masses with evolutionary tracks available, isochrones are interpolated in terms of

$$
\begin{aligned}
& \log L=\log \left(L\left(m_{\mathrm{i}}, \mu, \tau_{\mu}\right)\right) \\
& \log T_{\text {eff }}=\log \left(T_{\text {eff }}\left(m_{\mathrm{i}}, \mu, \tau_{\mu}\right)\right)
\end{aligned}
$$

where $m_{\mathrm{i}}$ is the initial mass for track $i, \mu$ the defined physical phase of evolution and $\tau_{\mu}$ the relative duration of this phase.

It is then possible to interpolate both in luminosity and temperature between two adjacent masses. For the interpolated masses the magnitudes and colors are then obtained by translating the theoretical luminosities and effective temperatures with tables of bolometric corrections and colors.

We supplemented these Padova isochrones by low mass stars in the mass range from $0.15-0.45 M_{\odot}$ from the calculations of Chabrier \& Baraffe (1997) that include a new description of the interior of low mass objects and use non-grey atmospheres (see Kurth et al. 1999 for details). The Padova group recently added low mass-stars to their isochrones themselves (Girardi et al. 2000) but did not compare their low mass stars to those of Chabrier \& Baraffe. The cumulative contribution of stars below $0.5 M_{\odot}$, however, to the integrated light is very small, anyway.

An important feature of the 1999 Padova isochrones is that they include a semi-analytical description of the thermal pulsing asymptotic giant branch (TP-AGB) phase (cf. Groenewegen \& de Jong 1993), which was not yet included in the earlier Padova tracks (cf. Bressan et al. 1993; Fagotto et al. 1994a, 1994b, 1994c).

TP-AGB stars are rare species in single burst populations, they are, however, very luminous and very cool (Lançon 1999). At ages between 0.1 and $1 \mathrm{Gyr}$, i.e. when the red supergiants have died already and the red giants are not important yet, TPAGB stars account for 25 to $40 \%$ of the bolometric light of an 

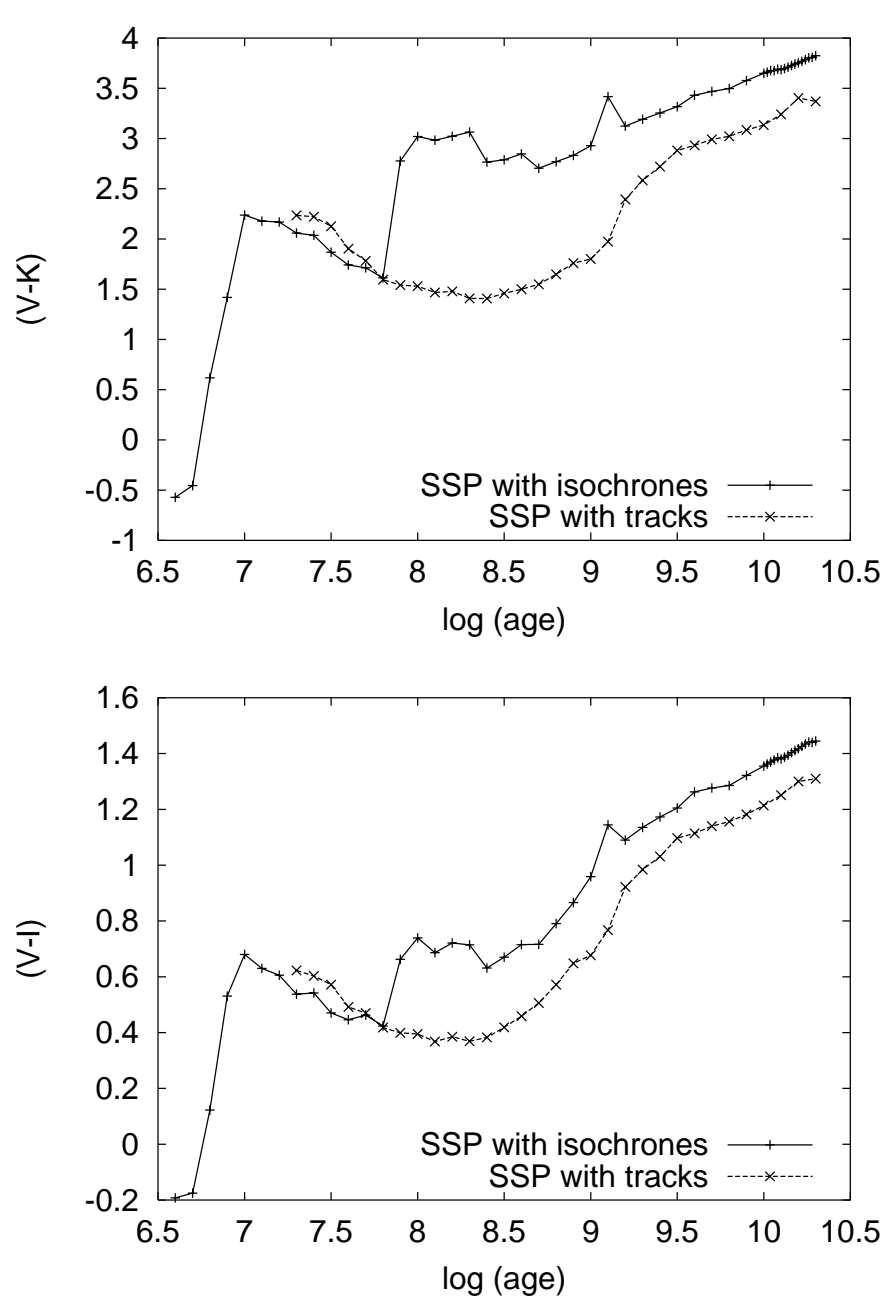

Fig. 1. Time evolution of $(V-K)$ and $(V-I)$ colors (as a function of logarithmic age [yr]) for SSPs based on isochrones, i.e. including the TP-AGB phase (solid lines), and based on stellar evolutionary tracks that did not yet include the TP-AGB phase (dashed lines), both for solar metallicity.

SSP and for 40 to $60 \%$ of the light emitted in the $K$-band (cf. Charlot 1996).

Inclusion of the TP-AGB phase has strong effects on some of the colors. It makes the broad band colors redder in the age range from $10^{8}$ to a few $10^{9} \mathrm{yr}$. This effect is strongest in $(V-K)$ and can amount to more than 1 mag in $(V-K)$ at solar metallicity as seen in Fig. 1, where we plot the $(V-K)$ color evolution for an SSP based on isochrones including the TP-AGB in comparison with an SSP from Kurth et al. (1999) based on the Padova stellar evolutionary tracks that did not include the TP-AGB phase. The effect is weaker at lower metallicities and for colors at shorter wavelengths.

The effect of the TP-AGB phase has been discussed by the Padova group before (Girardi et al. 1998) and the good agreement with $J-$ and $K-$ CMDs of resolved young open clusters is shown in Vallenari et al. (1999). Rich et al. (2001) find excellent agreement of the recent Padova isochrones with CMDs in $U B V$ and $I$ for the LMC cluster NGC 2121 which, at $[\mathrm{Fe} / \mathrm{H}]=-0.6$ and an age of $3.2 \mathrm{Gyr}$, is a very good test case for the importance of the TP-AGB phase.
The effect of the TP-AGB phase is also significant in $(V-I)$ at metallicities $\geq 0.5 Z_{\odot}$ and has important implications for the age determination on the basis of colors observed for bright star clusters in interacting and/or starburst galaxies. E.g. in our analysis of the sample of young star clusters in the merger remnant NGC 7252 which show a mean $(V-I)=0.83 \pm 0.25$ we derived a mean age of $1.37_{-0.8}^{+1.6} \mathrm{Gyr}$ assuming a metallicity $\approx 0.5 Z_{\odot}$ using SSP models based on Geneva stellar evolutionary tracks (Fritze - v. Alvensleben \& Burkert 1995) very similar to what is found with the models of Kurth et al. (1999) who use Padova tracks. Including the TP-AGB phase as in the Padova isochrones used here, however, results in a mean age of $8.7 \times 10^{8} \mathrm{yr}$, i.e. only $64 \%$ of the age obtained without the TP-AGB phase. Most young star cluster systems observed with HST, in fact, have ages in the range of $10^{8}$ to $10^{9} \mathrm{yr}$ and metallicities $\geq 0.5 Z_{\odot}$ where the effect of the TP-AGB phase on $(V-I)$ is strong. Hence, all their age determinations on the basis of $(V-I)$ in the literature are subject to this effect.

\subsection{Input physics: Spectra}

We use the library of model atmosphere spectra from Lejeune et al. (1997, 1998) which is based on model atmospheres from Kurucz (see e.g. Kurucz 1992), Fluks et al. (1994), and Bessell et al. (1989, 1991). This library comprises the full range of metallicities covered by the isochrones, is very complete in terms of stellar effective temperatures from $T_{\text {eff }}=$ 2800-47 $500 \mathrm{~K}$, and gravities, and has a long wavelength coverage from $90 \AA$ to $160 \mu \mathrm{m}$ with good spectral resolution. Its spectra have partly been corrected to provide good agreement with the observed colors of all types of stars from $U$ through $K$ (cf. Lejeune et al.). Stars with an effective temperature above $50000 \mathrm{~K}$ are described by pure black body spectra.

All the coolest AGB stars are long period variables which have different spectral type - temperature - color relations and, in particular, strongly increased molecular absorption features (e.g. $\mathrm{H}_{2} \mathrm{O}$ around 1.4 and $1.9 \mu \mathrm{m}, V O$ at $1.05 \mu \mathrm{m}, \ldots$ ) as compared to non-pulsating stars (Lançon 1999). Lançon \& Wood (1997) compiled an IR - library of cool star spectra that was combined with the one of Lejeune et al. (1998) that we use here and implemented into models by Fioc \& Rocca-Volmerange (1997). These models show that the inclusion of observed AGB spectra is very important for a description of specific spectral features in the near and mid-IR of intermediate age SSPs. Bressan et al. (1998) investigate in detail the effects of dust in the envelopes of AGB stars on the integrated IR properties of intermediate age SSPs. They consider both the absorption of light in the expanding dust shells of mass-losing stars at optical wavelengths and its thermal reemission in the IR. Differences with respect to a standard SSP without these dust absorption and emission features appear beyond a few $\mu \mathrm{m}$ where the contribution of the brightest AGB stars to the integrated light of the stellar population is large. Bressan et al. find a decrease of the flux between 1 and $3 \mu \mathrm{m}$ because the brightest stars are heavily obscured by their circumstellar envelopes and thermal dust emission at longer wavelengths $\gtrsim 4 \mu \mathrm{m}$, reaching an order of magnitude at $10 \mu \mathrm{m}$ over the pure photospheric models. 
The UV, optical and NIR regions we are studying in this paper are not affected by the expanding dust shells surrounding TP-AGB stars. We caution, however, that our models do not include the above-mentioned empirical AGB spectra yet, nor the absorption and reemission of light by their dusty envelopes, and, hence, should not be used for the interpretation of fluxes or spectral features of intermediate age star clusters beyond the $K$-band. In stellar populations with extended SFHs the AGB star features discussed above (molecular absorption bands, extinction and reemission by surrounding dust shells) are strongly overwhelmed by the emission of coexisting red supergiant and giant stars.

\subsection{Isochrone synthesis model}

Our isochrone synthesis code for SSPs starts from a gas cloud of given mass and metallicity and then forms a population of stars with a given SFR during the first timestep of the calculation. It calculates a variety of observables at any evolutionary timestep, typically $4 \times 10^{6} \mathrm{yr}$ :

- masses in gas and stars;

- ejection rates of gas and metals by dying stars;

- spectra from $90 \AA$ to $160 \mu \mathrm{m}$;

- luminosities, mass-to-light - ratios, and colors in various filters;

- evolutionary and cosmological corrections including the effect of attenuation by intergalactic hydrogen.

Ejection rates of gas and metals by dying stars are calculated from the individual stellar gas and heavy element yields for the respective isochrone metallicities.

Every star of mass $m$ on an isochrone of age $t$ and metallicity $Z$ is attributed a spectrum $\left(S_{*}(\lambda, m)\right)$ from Lejeune et al's spectral library, appropriate for its effective temperature $T_{\text {eff }}$ and surface gravity $g$. Because our model is a 1-zone model without any spatial resolution, spectra of all stars along an isochrone of given metallicity $Z$ can be summed up, weighted with the IMF $\Phi(m)$, to give the integrated isochrone spectrum $\left(S_{\text {iso }}(\lambda, t, Z)\right)$, i.e. the spectrum of an SSP of metallicity $Z$ at age $t$

$S_{\text {iso }}(\lambda, t, Z)=\int_{m_{1}}^{m_{\mathrm{u}}} S_{*}(\lambda, m, t, Z) \cdot \Phi(m) \cdot \mathrm{d} m$.

Alternatively, the code can also model arbitrary stellar populations with extended or complex star formation histories, such as galaxies. For the evolution of a galaxy with a star formation rate extended in time $(S F R(t))$, isochrones are followed as a function of time and multiplied with the star formation rate at the formation time $t_{\mathrm{i}}$ of the respective isochrone, $\operatorname{SFR}\left(t_{\mathrm{i}}\right)$. So the integrated spectrum of a galaxy $\left(S_{\text {gal }}(\lambda, t, Z)\right)$ at time $t$ is calculated via Eq. (2)

$S_{\text {gal }}(\lambda, t, Z)=\int_{0}^{t} S_{\text {iso }}\left(\lambda,\left(t-t_{\mathrm{i}}\right), Z\right) \cdot S F R\left(t_{\mathrm{i}}\right) \cdot \mathrm{d} t_{\mathrm{i}}$.

To account for the fact that, in general, the stellar population of a galaxy is comprised of subpopulations of different metallicities, an additional integration over all the metallicities can be performed. Here, however, we only present results for our SSP models.

\subsection{Filter systems}

Several filter systems are implemented in our evolutionary synthesis code, as e.g. Johnson $U B V R I$ (cf. Lamla 1982), J H K L (Bessel \& Brett 1988), gr (Thuan \& Gunn 1976), $U^{+} J^{+} F^{+} N^{+}$(Kron 1980; Koo 1985), HST F300W ... $F 814 W$, Washington $C M T 1 T 2$ (Harris \& Canterna 1979), and Strömgren $u v b y$ (cf. Lamla 1982). Results for all of them are presented in our electronic tables.

Calibrations for the Washington filters were done with colors for stars in Gunn \& Stryker's (1983) spectral library (Geisler 2000 priv. comm.). The Johnson, Thuan \& Gunn and Bessel \& Brett filter systems were calibrated with information from Landolt-Börnstein VI/2b. The HST-Filters were calibrated with Kurucz's Vega model as described on the HST home page. The calibration of Strömgren photometry was done according to Gray (1998).

With the time evolution of SSP spectra as presented here, any other filter system within the long wavelength range of our spectra from $90 \AA$ through $160 \mu \mathrm{m}$ is readily applicable. Integrating over the spectra with filter transmission and detector response functions allows to study both the time evolution and the metallicity dependence of any arbitrary filter system.

\subsection{Cosmological models}

As outlined in Sect. 1, our SSP results are not only intended for use in the interpretation of star cluster data but also for combination with any kind of dynamical galaxy formation and/or evolution model. For convenient use in this second respect, we also calculate evolutionary and cosmological corrections.

Spectra are redshifted to $\lambda^{\prime}(z)=\lambda \cdot(1+z)$ and fluxes are decreased by $F_{\lambda}^{\prime}\left(\lambda^{\prime}(z), z\right)=F_{\lambda}\left(\lambda^{\prime}(z), 0\right) /(1+z)$.

For galaxies evolutionary and cosmological corrections are conventionally given in terms of magnitude differences, which are normalized to the average luminosity of locally observed galaxies of the respective type. SSPs, however, do not have such an absolute magnitude scale. We therefore chose to give their evolutionary and cosmological corrections in terms of luminosity ratios rather than magnitude differences and call them $\epsilon_{\lambda}$ and $\kappa_{\lambda}$, respectively, not to be confused with the $e_{\lambda}$ and $k_{\lambda}$ conventionally given for galaxies:

$\epsilon_{\lambda}=\frac{L_{\lambda}(z, t(z))}{L_{\lambda}\left(z, t_{0}\right)}$

$\kappa_{\lambda}=\frac{L_{\lambda}\left(z, t_{0}\right)}{L_{\lambda}\left(0, t_{0}\right)}$

with $t(z)$ being the age of a galaxy observed at redshift $z$ and $t_{0}$ the age of a galaxy at $z=0$. Table 1 gives the ages $t(z)$ of a stellar population formed at redshift $z_{\mathrm{f}}=5.0$ and the Bolometric Distance Modulous $(B D M(z))$ for two specific cosmological models $\left(H_{0}, \Omega_{0}, \Lambda_{0}\right)=(75,0.1,0.9)$ and $(65,0.1,0)$ we chose to present here. With the large number of spectra given for every $\operatorname{SSP}(Z)$ in short time intervals any arbitrary cosmological model can easily be applied. 
Table 1. Evolution time $t$ and $B D M$ as a function of redshift $z$ with the cosmology $H_{0}=75, \Omega_{0}=0.1, \Lambda_{0}=0.9$ on the left, and with $H_{0}=65$, $\Omega_{0}=0.1, \Lambda_{0}=0$ on the right hand side, respectively.

\begin{tabular}{cccccc}
\hline \hline$z$ & $t[\mathrm{Gyr}]$ & $B D M$ & $z$ & $t[\mathrm{Gyr}]$ & $B D M$ \\
\hline 0.0 & 15.12 & 36.512 & 0.0 & 11.92 & 31.15 \\
0.5 & 9.932 & 42.487 & 0.5 & 6.957 & 42.267 \\
1.0 & 6.720 & 44.375 & 1.0 & 4.535 & 44.130 \\
1.5 & 4.632 & 45.543 & 1.5 & 3.115 & 45.305 \\
2.0 & 3.222 & 46.377 & 2.0 & 2.189 & 46.179 \\
2.5 & 2.236 & 47.019 & 2.5 & 1.542 & 46.877 \\
3.0 & 1.522 & 47.536 & 3.0 & 1.065 & 47.461 \\
3.5 & 0.988 & 47.968 & 3.5 & 0.702 & 47.961 \\
4.0 & 0.579 & 48.337 & 4.0 & 0.417 & 48.400 \\
4.5 & 0.258 & 48.659 & 4.5 & 0.188 & 48.790 \\
5.0 & 0.0 & 48.945 & 5.0 & 0.0 & 49.141 \\
\hline
\end{tabular}

\section{Spectral evolution of SSPs for 5 different metallicities}

We present the time evolution of SSP spectra of 5 metallicities from $4 \times 10^{6} \mathrm{yrs}$ (which is the youngest isochrone) up to $14 \mathrm{Gyr}$ with a Salpeter and a Scalo IMF, respectively. The initial stellar mass is $1.6 \times 10^{9} M_{\odot}$ for both IMFs.

\subsection{Time evolution of spectra}

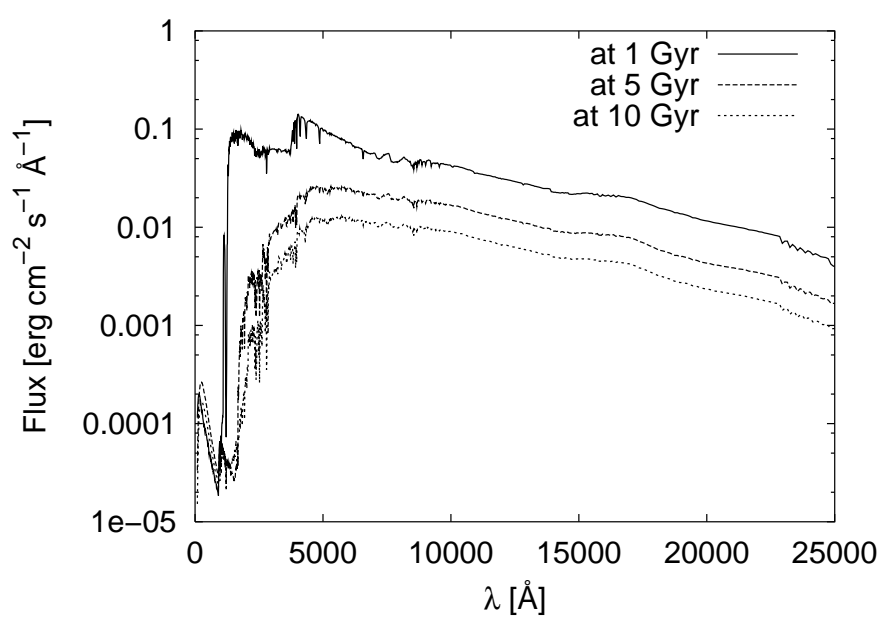

Fig. 2. Spectrum in terms of flux $F_{\lambda}$ as a function of wavelength $\lambda$ at 3 different times for an SSP of solar metallicity and Salpeter IMF.

In Fig. 2 we present the spectral evolution of an SSP with solar metallicity and Salpeter IMF at 3 different ages of 1, 5, and $10 \mathrm{Gyr}$, respectively. The flux decreases with time as stars of lower and lower masses die with the strongest effect seen between 1500 and $5000 \AA$. Note the small rise of the flux below $1000 \AA$ due to very hot stars on the white dwarf cooling sequence.

The 1 Gyr spectrum shows clearly the Balmer, Paschen, and Bracket series of hydrogen absorption lines. Note the almost unchanging flux at $\lambda \lesssim 1000 \AA$ and the strong time evolution in the $4000 \AA$ break.
The spectral evolution of an SSP with a Scalo IMF looks very similar to the one with a Salpeter IMF plotted here, because, at $1 \mathrm{Gyr}$, most of the high mass stars that were less numerous in an SSP with Scalo IMF, have already died. A small difference in absolute flux would be seen due to the higher number of low mass stars in the Scalo IMF, which dominate the flux at higher ages.

\subsection{Metallicity effects on the spectral evolution}
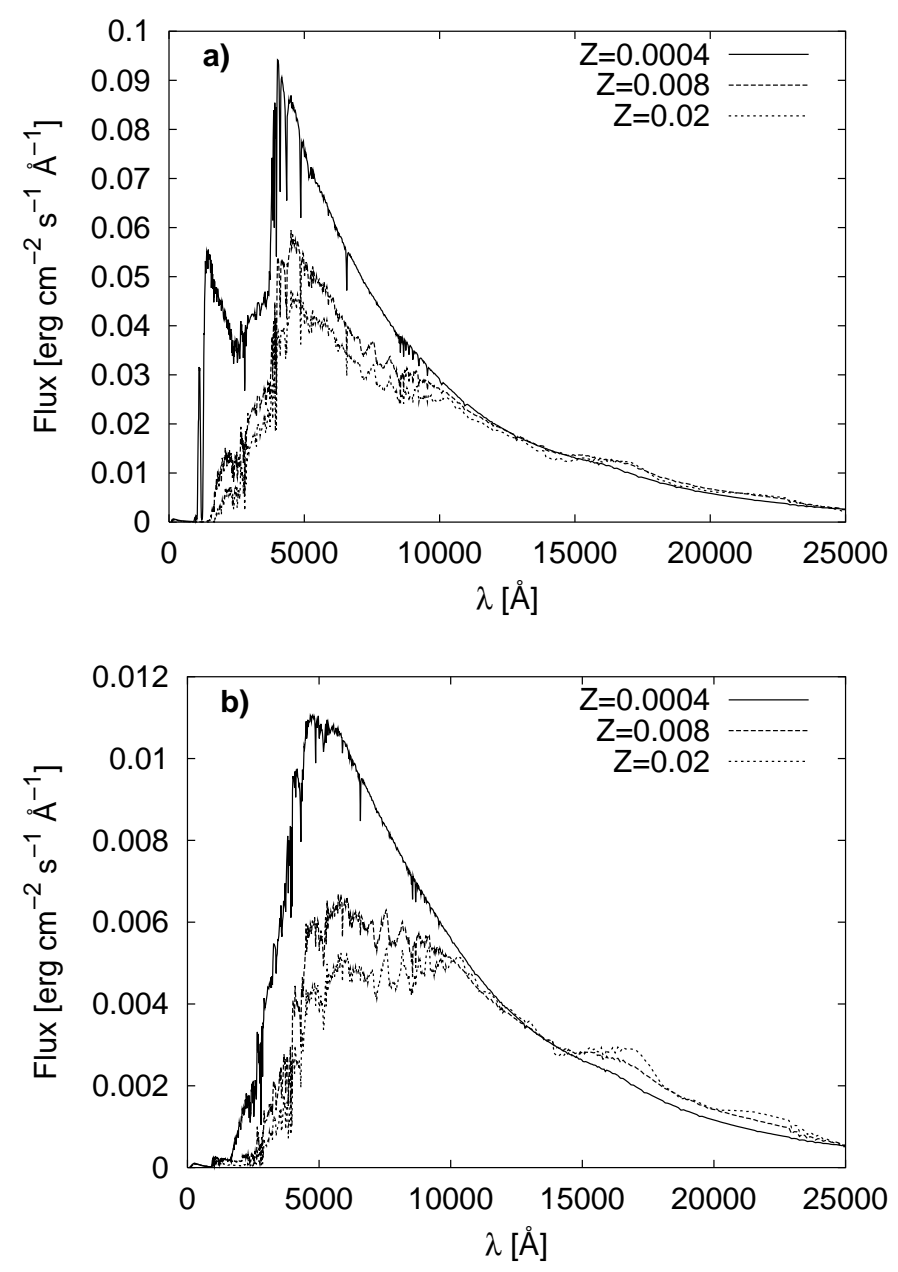

Fig. 3. Flux $F_{\lambda}$ as a function of wavelength for SSPs with Salpeter IMF and 3 different metallicities at ages of a) $1 \mathrm{Gyr}$ and b) $10 \mathrm{Gyr}$. $\left(Z=0.0004=0.02 Z_{\odot}, Z=0.008=0.4 Z_{\odot}, Z=0.02=Z_{\odot}.\right)$

In Figs. 3a and 3b, we show spectra for SSPs of 3 different metallicities at ages of 1 and $10 \mathrm{Gyr}$, respectively, all with a Salpeter IMF. Enormous differences are seen both at young and old ages between SSPs of different metallicities. As compared to a solar metallicity SSP, the spectrum of a low metallicity SSP shows significant differences both in continuum slope and absorption line strengths. Over the $U V$ and optical wavelength range the continuum spectrum of a low metallicity SSP has stronger flux and the maximum at shorter wavelengths as compared to a solar metallicity SSP. This is a consequence of stars - on average - being brighter and hotter at lower metallicity. 
Note the very strong $U V$ flux at $1000 \AA \lesssim \lambda \lesssim 2000 \AA$ of the 1 Gyr very low metallicity $\left(Z=0.02 Z_{\odot}\right)$ SSP. Not only the metal absorption lines are weaker - as expected - in low metallicity SSPs of all ages, but also the Balmer absorption lines, most prominent in the 1 Gyr old SSPs, are visibly stronger at low metallicities (cf. Kurth et al. 1999 for a quantitative analysis of absorption features).

At young ages $\sim 1 \mathrm{Gyr}$, the near infrared (NIR) continuum is largely metallicity independent while at older ages, e.g. at 10 Gyr as shown in Fig. 3b, it is visibly affected by metallicity effects. E.g. in the $1.7 \mu \mathrm{m}$ bump in the $H$-band, and at $2.2 \mu \mathrm{m}$ in the $K$-band a $10 \mathrm{Gyr}$ old $Z=0.02 Z_{\odot} \mathrm{SSP}$ only shows $\sim \frac{2}{3}$ of the flux of a solar metallicity SSP.

\subsection{Comparison with earlier models}

We compare our results with those from Bruzual \& Charlot's (1993) models (hereafter BC) as obtained from the CD-ROM accompanying Leitherer et al. (1996). In Fig. 4 we show a comparison of spectra from $1000 \AA$ to $25000 \AA$ a), a more detailed view from $5000 \AA$ to $10000 \AA$ b) and a view from 0 to $2000 \AA$ c) for SSPs with solar metallicity and Salpeter IMF. Both models show good agreement from the $U V$ to $\sim 7000 \AA$. Between $7000 \AA$ and $12000 \AA$, however, BC's spectrum shows significantly less flux than ours. The same happens again in the $H$ - and $K$-band regions. This probably is due to the TPAGB phase which involves stars with an effective temperature around $3000 \mathrm{~K}$. The comparison in the wavelength range from $90 \AA$ to $2000 \AA$ shows a strong difference between the models. In this wavelength region very hot stars dominate the flux. These are stars on the white dwarf cooling sequence. We do not know in detail how theses stars are handled in BC's models but we suspect that the flux difference is due to a different treatment of white dwarfs.

\section{Luminosity evolution, colors, and mass-to-light ratios}

\subsection{Luminosity evolution}

In Fig. 5 we present the time evolution of $V-, J-$, and $K$-band absolute magnitudes of SSPs with 3 different metallicities. Again, the metallicity effects are discussed on the example of a Salpeter IMF.

All over the time evolution, SSPs are brighter at lower metallicities in all bands $U, \ldots, I$ (Fig. 5a). The difference in $M_{V}$ between a solar metallicity SSP and one with $0.02 Z_{\odot}$ increases from $\lesssim 0.5 \mathrm{mag}$ at few $10^{8}$ yr to $\sim 1 \mathrm{mag}$ at $5-14 \mathrm{Gyr}$.

As seen in Fig. $5 \mathrm{~b}$, the time evolution of $M_{J}$ does not show any significant metallicity dependence, as could already be expected from the spectra in Figs. 3a and 3b. The spectra also indicate what is clearly found for the $H$ - and $K$-band evolution: the trend of luminosity with metallicity is reversed in the near infrared spectral region as compared to optical wavelengths with the higher metallicity SSPs being brighter in $H$ and $K$ (cf. Fig. 5c), although the luminosity differences are not as strong as they are at shorter wavelengths.
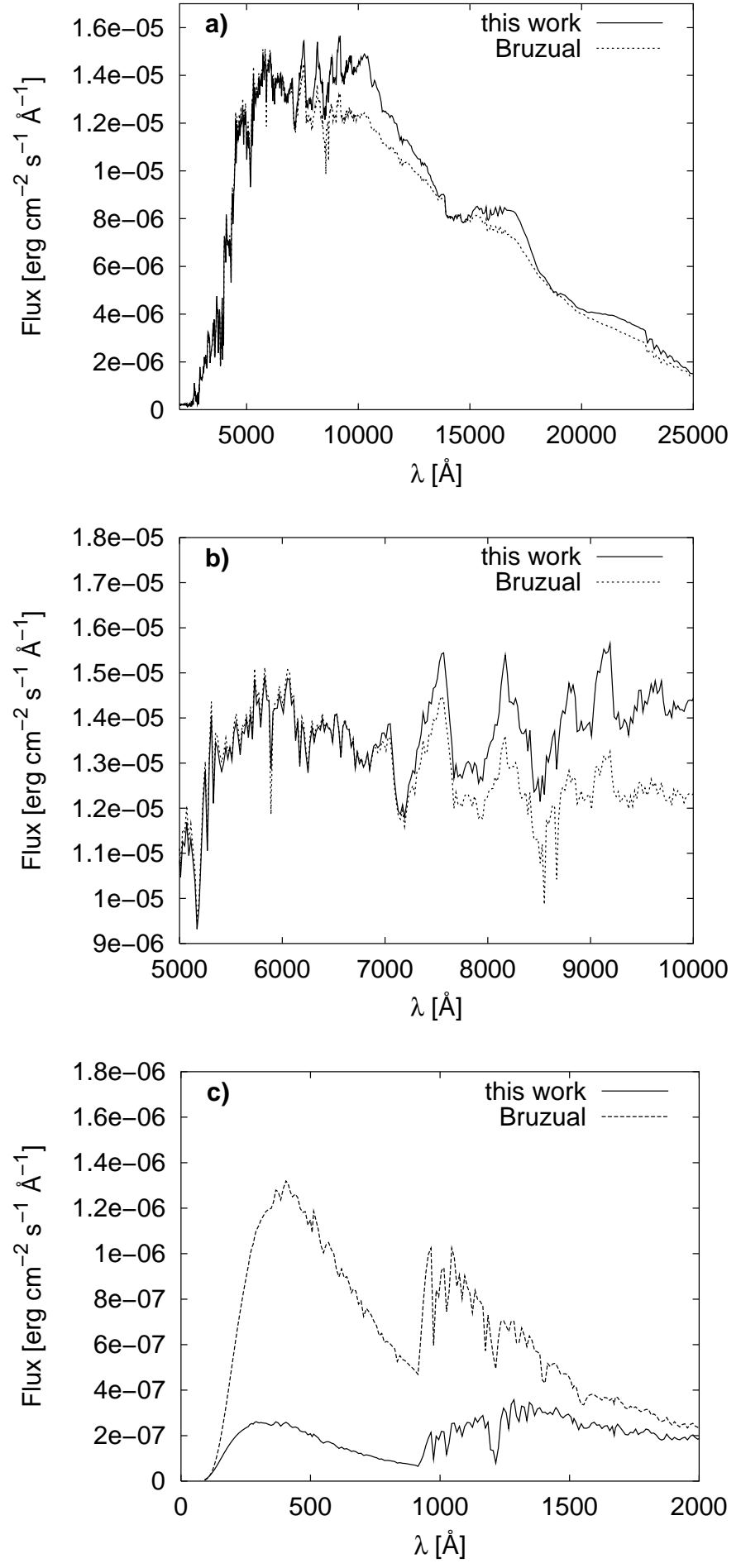

Fig. 4. Spectra in terms of flux as a function of wavelength. Comparison between our model and the model from Bruzual \& Charlot at 12 Gyr with Salpeter IMF and solar metallicity in 3 different wavelength ranges.

\subsection{Color evolution}

The $\left(V-I_{\mathrm{c}}\right)$ and $(B-V)$ color evolution for SSPs of various metallicities with a Salpeter IMF is plotted in Fig. 6. Note that $I_{\mathrm{c}}$ now is the Cousins $I$ for the purpose of comparison with data for M 31 clusters. On the right hand side of Fig. 6 we give the median colors with their $1 \sigma$ dispersions of M 31 clusters 

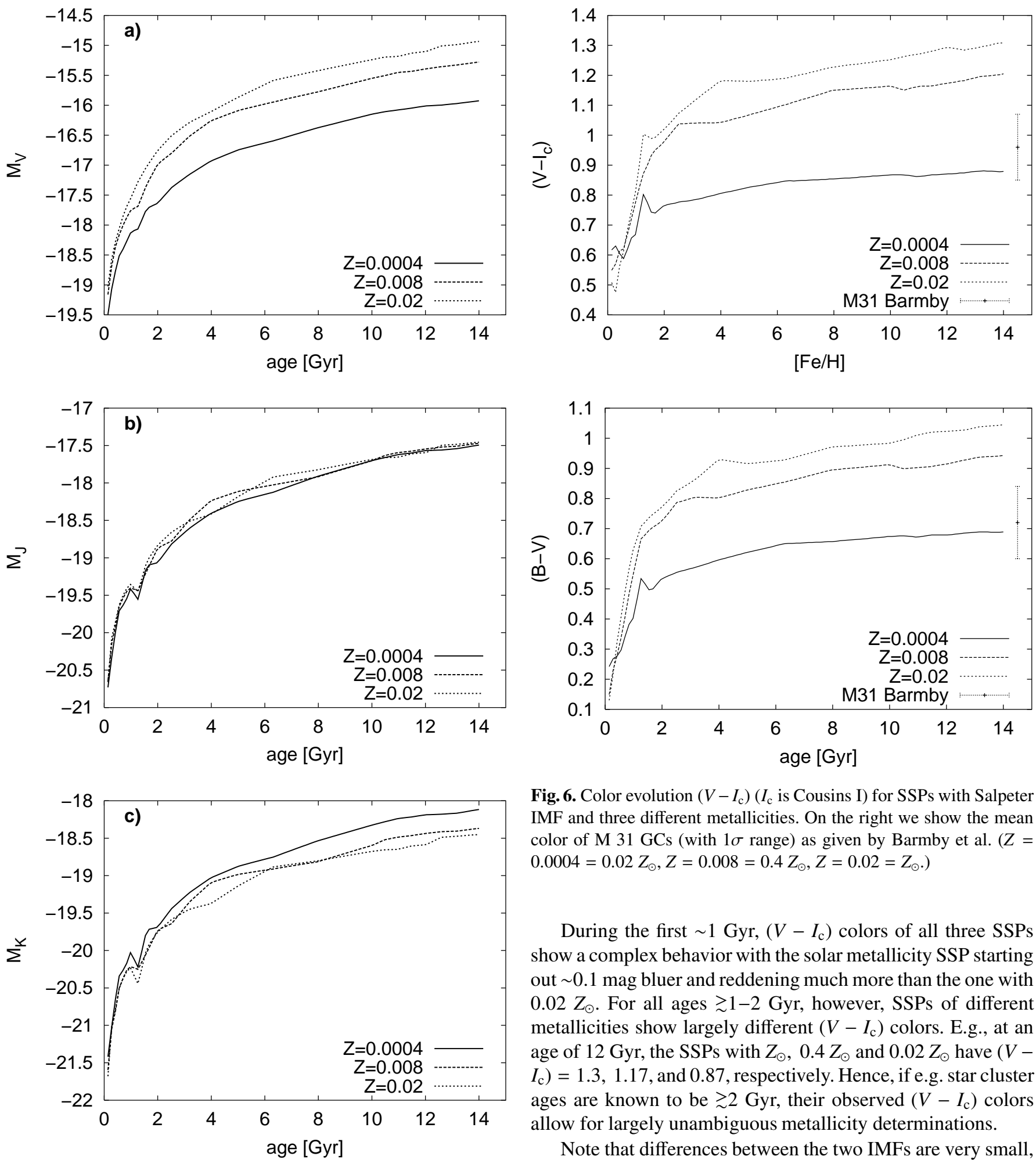

Fig. 6. Color evolution $\left(V-I_{\mathrm{c}}\right)\left(I_{\mathrm{c}}\right.$ is Cousins I) for SSPs with Salpeter IMF and three different metallicities. On the right we show the mean color of M 31 GCs (with $1 \sigma$ range) as given by Barmby et al. ( $Z=$ $0.0004=0.02 Z_{\odot}, Z=0.008=0.4 Z_{\odot}, Z=0.02=Z_{\odot}$.)

During the first $\sim 1 \mathrm{Gyr},\left(V-I_{\mathrm{c}}\right)$ colors of all three SSPs show a complex behavior with the solar metallicity SSP starting out $\sim 0.1 \mathrm{mag}$ bluer and reddening much more than the one with $0.02 Z_{\odot}$. For all ages $\gtrsim 1-2 \mathrm{Gyr}$, however, SSPs of different metallicities show largely different $\left(V-I_{\mathrm{c}}\right)$ colors. E.g., at an age of $12 \mathrm{Gyr}$, the SSPs with $Z_{\odot}, 0.4 Z_{\odot}$ and $0.02 Z_{\odot}$ have $(V-$ $\left.I_{\mathrm{c}}\right)=1.3,1.17$, and 0.87 , respectively. Hence, if e.g. star cluster ages are known to be $\gtrsim 2 \mathrm{Gyr}$, their observed $\left(V-I_{\mathrm{c}}\right)$ colors allow for largely unambiguous metallicity determinations.

Note that differences between the two IMFs are very small, as already mentioned above, and therefore not shown here. In particular, they almost do not affect age and/or metallicity determinations at ages $\gtrsim 4 \times 10^{8} \mathrm{yr}$. $J-\mathbf{b}$ ), and $K$-bands c) for SSPs with Salpeter IMF and 3 different metallicities. $\left(Z=0.0004=0.02 Z_{\odot}, Z=0.008=0.4 Z_{\odot}\right.$, $Z=0.02=Z_{\odot}$.)

which have a mean metallicity $[\mathrm{Fe} / \mathrm{H}]=-1.2$ as determined by Barmby et al. (2000). We note that observed integrated GC colors are in good agreement with our models for the corresponding metallicity at ages $12-14 \mathrm{Gyr}$.

\subsection{Mass-to-light ratios}

In Fig. 7 we show the time evolution of the total mass in stars, $M$, in SSPs with Salpeter and Scalo IMFs and three different metallicities. In all cases, SSPs started out with an initial stellar mass $M=1.6 \times 10^{9} M_{\odot}$ as mentioned above. 

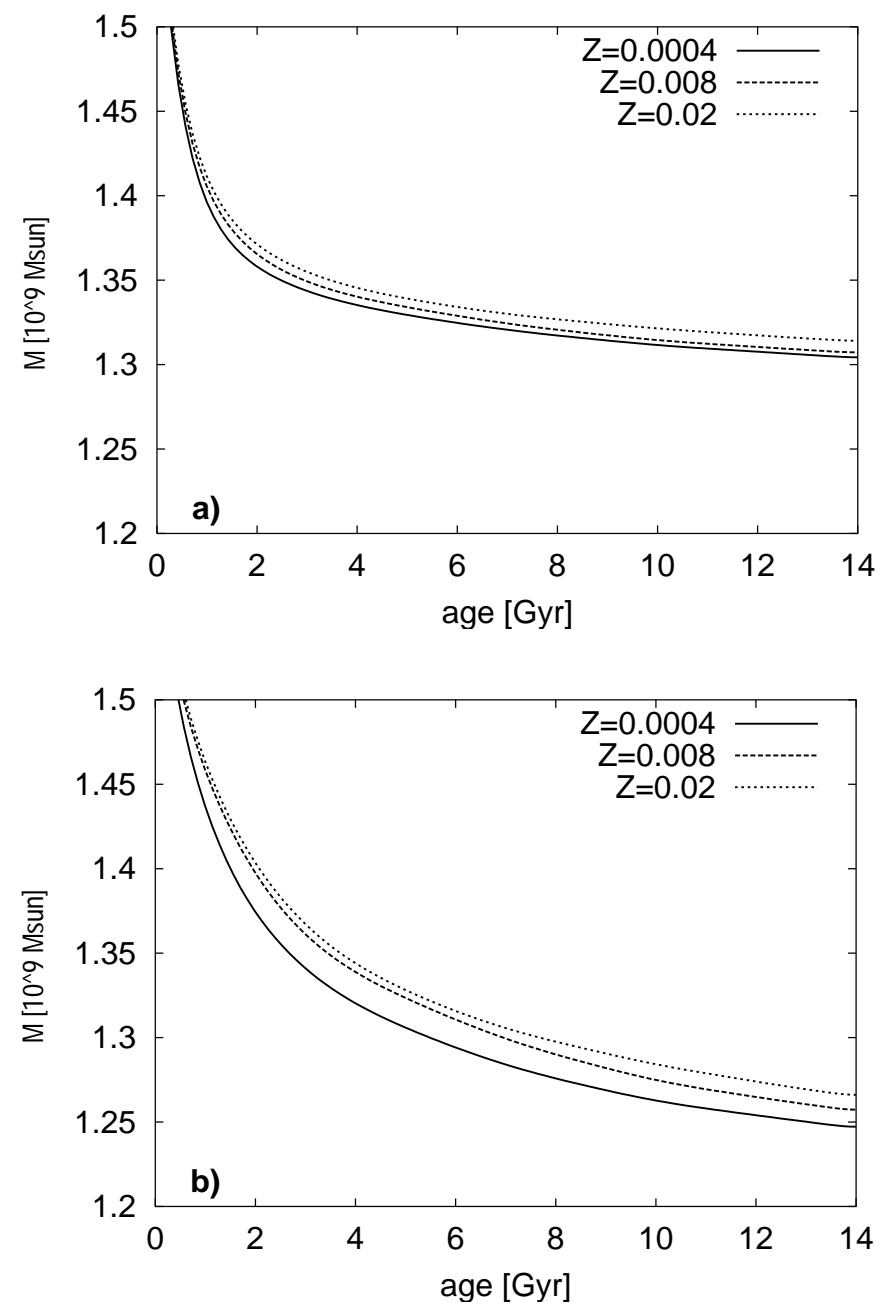

Fig. 7. Time evolution of the total stellar mass $M$ in units of $10^{9} M_{\odot}$ for SSPs with a) Salpeter and b) Scalo IMF and three different metallicities. $\left(Z=0.0004=0.02 Z_{\odot}, Z=0.008=0.4 Z_{\odot}, Z=0.02=Z_{\odot}.\right)$

The total stellar mass $M$ decreases as stars lose mass by stellar winds and die after ejecting mass either in a PN or in a SN event. With a Salpeter and a Scalo IMF, SSPs lose $\sim 19 \%$ and $\sim 23 \%$, respectively, of their initial mass until an age of 1214 Gyr. The bulk of the mass loss occurs during the first $\sim 2 \mathrm{Gyr}$ in case of a Salpeter IMF due to its larger number of high mass stars, while mass loss occurs more steadily with a Scalo IMF.

While for an SSP with Salpeter IMF the metallicity dependence of the mass loss is very small, it is slightly stronger in the case of a Scalo IMF (23\% for $Z_{\odot}$ and $20 \%$ for $0.02 Z_{\odot}$ ).

Although the difference in mass loss is small between SSPs with Salpeter and Scalo IMFs, the differences in mass-to-light ratios $M / L_{\lambda}$, with $\lambda$ denoting any filter band, are much larger because of the luminosity differences.

Figure 8 shows the time evolution of $V$-band mass-to-light ratios $M / L_{V}$ of SSPs with both IMFs and three metallicities. The mass is the time evolving stellar mass of the SSP with mass loss included as depicted in Fig. 7.

Note the difference of more than a factor of 2 in $M / L_{V}$ at all times and for all metallicities between a Salpeter and a Scalo IMF and also the strong metallicity dependence in both cases.
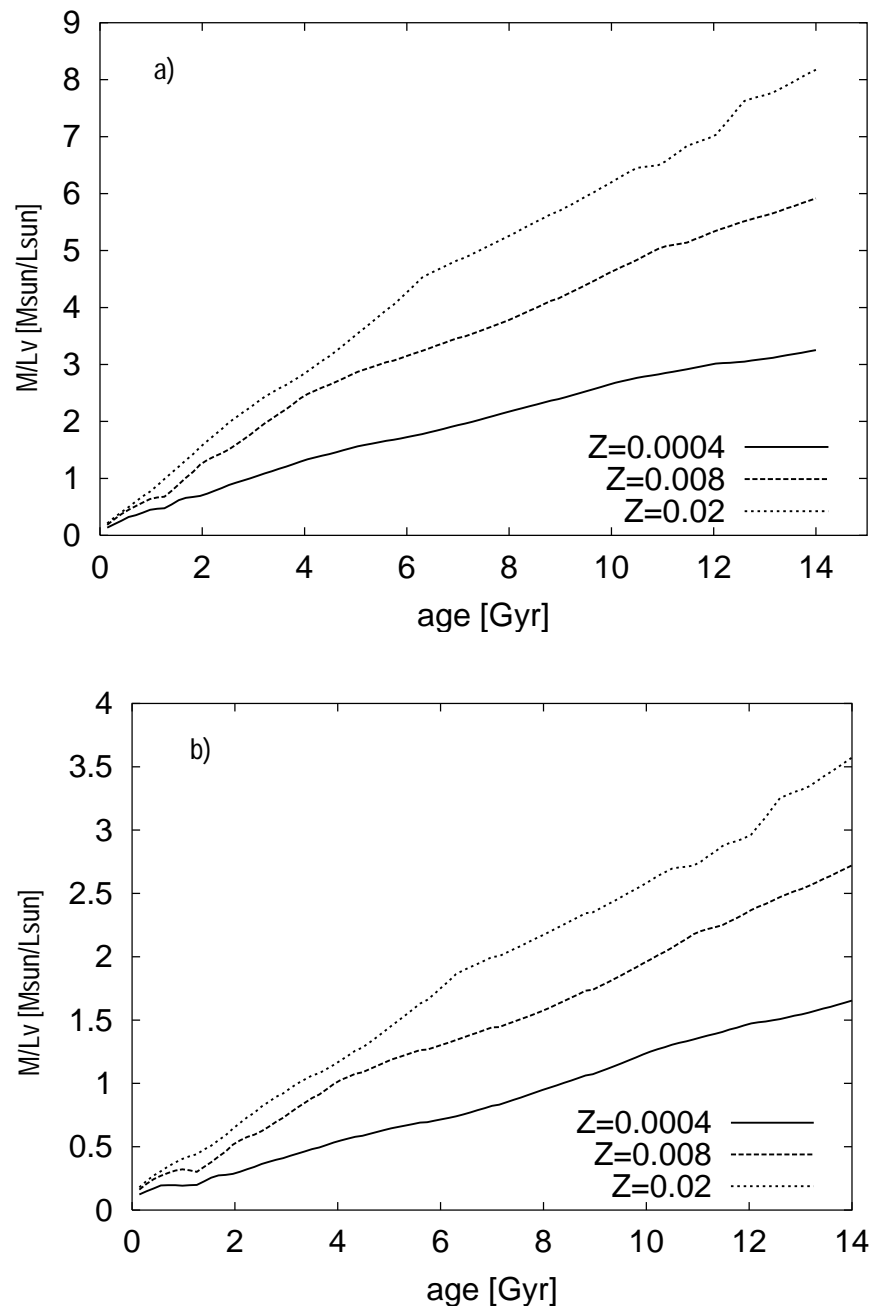

Fig. 8. Time evolution of $M / L_{V}$ in solar units for SSPs with Salpeter (top panel) and Scalo (bottom panel) IMFs and three different metallicities. $\left(Z=0.0004=0.02 Z_{\odot}, Z=0.008=0.4 Z_{\odot}, Z=0.02=Z_{\odot}\right.$. $)$

At $10 \mathrm{Gyr}$, a Salpeter SSP has $M / L_{V} \sim 12, \sim 9, \sim 5$ in solar units for $Z=Z_{\odot}, 0.4 Z_{\odot}$, and $0.02 Z_{\odot}$, respectively. For an extensive discussion of $M / L$-values (and their uncertainties) both theoretically derived by various groups using different sets of input physics and in comparison with observationally derived $M / L$-values obtained for GCs using various methods see Fritze - v. Alvensleben (2000).

\section{Theoretical calibrations of colors in terms of metallicity and comparison with GC data}

When spectroscopic abundance determinations are inaccessible or exceedingly time-consuming, like e.g. for GCs in external galaxies, broad band colors in combination with empirical calibrations with well known abundances are used (cf. Couture et al. 1990).

Clearly, some colors and color systems (like e.g. the Strömgren system) are better suited for this purpose than others. It is important to note, however, that in particular for optical and NIR colors the relations between color and metallicity are more or less age-dependent. This is seen 

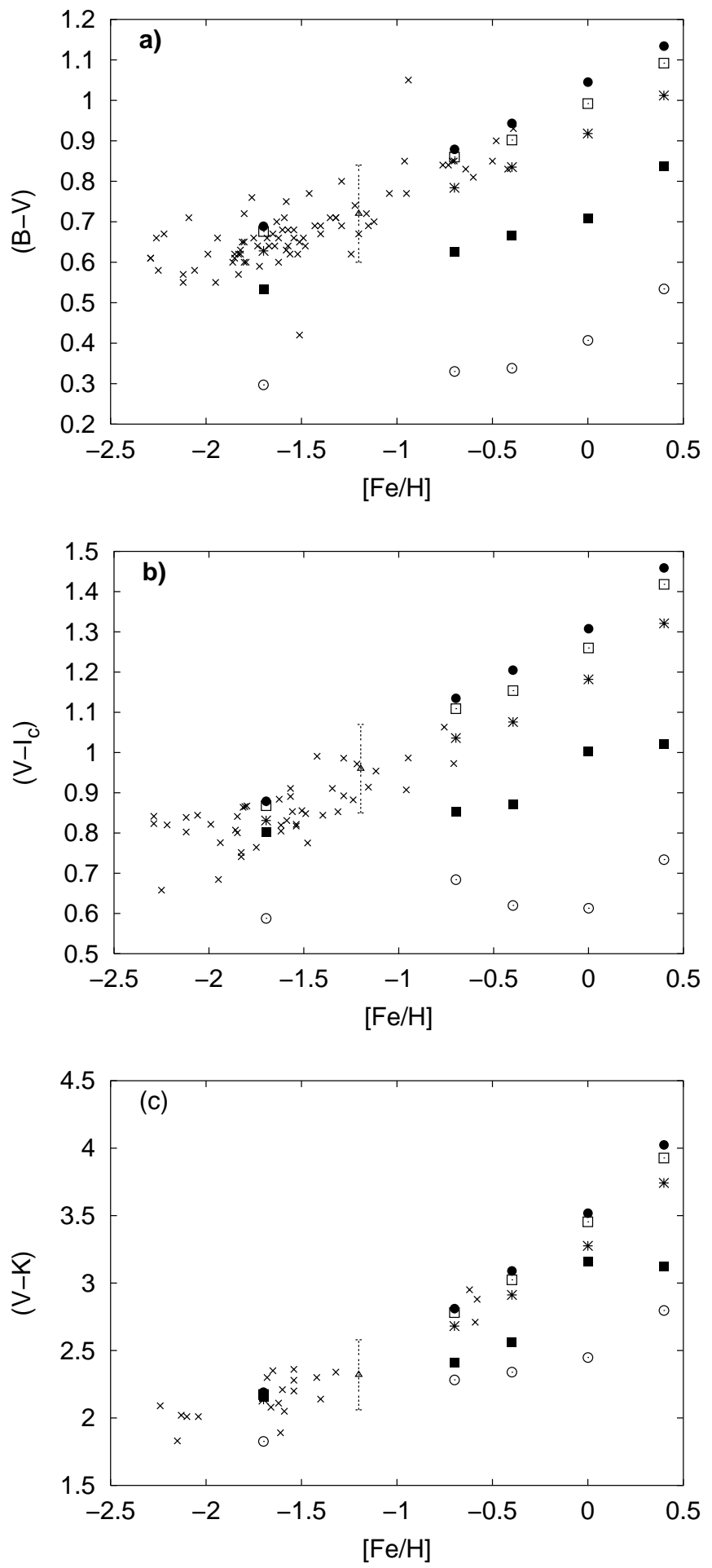

Fig. 9. a) $(B-V)$, b) $\left(V-I_{\mathrm{c}}\right)\left(I_{\mathrm{c}}\right.$ is Cousins $\left.\mathrm{I}\right)$ and c) $(V-K)$ color vs. metallicity $[\mathrm{Fe} / \mathrm{H}]$ for SSPs with Salpeter IMF at various ages. $0.5 \mathrm{Gyr}$ (open circles), $1 \mathrm{Gyr}$ (filled squares), $5 \mathrm{Gyr}$ (stars), $10 \mathrm{Gyr}$ (open squares), and 14 Gyr (filled circles). Crosses in a) and b) are Milky Way GC data from Harris' catalogue. In c) the crosses are M 31 GC data from Brodie \& Huchra. Also included in all three figures are the mean M 31 GC data and their $1 \sigma$ ranges from Barmby et al.

in Figs. 9a and 9b, where we plot theoretical calibrations obtained from our SSP models at various ages for $(B-V)$ (Fig. 9a), $\left(V-I_{\mathrm{c}}\right)($ Fig. 9b) and $(V-K)($ Fig. 9c) versus $[\mathrm{Fe} / \mathrm{H}]$. We also include observations of GCs in the Milky way from the GC catalogue compiled by W. E. Harris (1996) (we only use clusters with $E(B-V)<0.4)$ and median colors of GCs in M 31 from Barmby et al. (2000) in Fig. 9 which show good agreement with our models at 10-14 Gyr and low metallicities. In Fig. 9c observations from Brodie \& Huchra (1990) are included which also show very good agreement with our models. In this low metallicity regime empirical calibrations can be used with good accuracy. In all three colors it is seen that at young ages the theoretical calibrations are significantly different from what they are at old ages. Strong changes are seen between 0.5 and 1 Gyr and between 1 and 5 Gyr, smaller changes at older ages, e.g. between 5 and 14 Gyr. Note that theoretical calibrations can reach the highest metallicities for which stellar evolutionary tracks or isochrones are available, i.e. $2.5 \mathrm{Z}_{\odot}$ in our case, while observational calibrations are restricted to the metallicity range of Milky Way GCs, i.e. to $Z \lesssim 0.3 Z_{\odot}$.

Over the metallicity range of the GC sample $-2 \lesssim$ $[\mathrm{Fe} / \mathrm{H}] \lesssim-0.5$ the agreement between our theoretical calibrations at $12 \mathrm{Gyr}$ and the empirical ones from Couture et al. (1990) is very good, i.e. better than $0.05 \mathrm{mag}$ both in $(B-V)$ and $\left(V-I_{\mathrm{c}}\right)$.

Note, however, that extrapolations from the approximately linear observational relation at $[\mathrm{Fe} / \mathrm{H}] \lesssim-0.5$ to higher metallicities would produce grossly misleading results since towards higher metallicities the theoretical relations become significantly steeper. By extrapolating the empirical linear relation, a $10-14$ Gyr old cluster with e.g. $(B-V)=1.1$ would be ascribed a metallicity $Z_{\text {extrap }} \approx 4.4 Z_{\odot}$ while our model shows it to only have $Z \approx 2.5 Z_{\odot}$.

For $\left(V-I_{\mathrm{c}}\right)$, the deviation from the linear relation valid in the low metallicity region is even stronger. E.g. an old cluster with $\left(V-I_{\mathrm{c}}\right)=1.2$ would be ascribed $Z_{\text {extrap }} \approx 3.0 Z_{\odot}$ while our models indicate solar metallicity, i.e. three times lower! We stress that in particular for investigations of intermediate age cluster populations or of clusters with metallicities that might exceed those of the Milky Way or M 31 GCs (on which the empirical calibrations are based) it is very dangerous to use empirical calibrations. Our models clearly show the age dependence as well as the strong discrepancies from the empirical linear color - metallicity relations for $[\mathrm{Fe} / \mathrm{H}] \gtrsim-0.5$.

In Figs. 10a and $10 \mathrm{~b}$ we present analogues to Figs. 9a$\mathrm{c}$ for the Strömgren color indices $m_{1}=(v-b)-(b-y)$ and $c_{1}=(u-v)-(v-b)$. For the particularly metal-sensitive index $m_{1}$ it is seen that it allows to well separate clusters in terms of metallicities and ages. It allows for a better metallicity separation on the basis of colors than the Johnson filter system does. But it has a smaller absolute difference, which makes it fragile to observational uncertainties.

The age-sensitive Strömgren color index $c_{1}$ shows a completely different behavior. While it is, indeed, relatively constant at given age for all $[\mathrm{Fe} / \mathrm{H}] \lesssim-0.5$, it starts changing significantly towards higher metallicities, where it allows for metallicity separations nearly as good as $m_{1}$. Due to its changing behavior, it is important to well know the metallicity of the cluster, which should be $<-0.5$ in $[\mathrm{Fe} / \mathrm{H}]$. Above this metallicity, even $m_{1}$ shows a better age separation than $c_{1}$. 

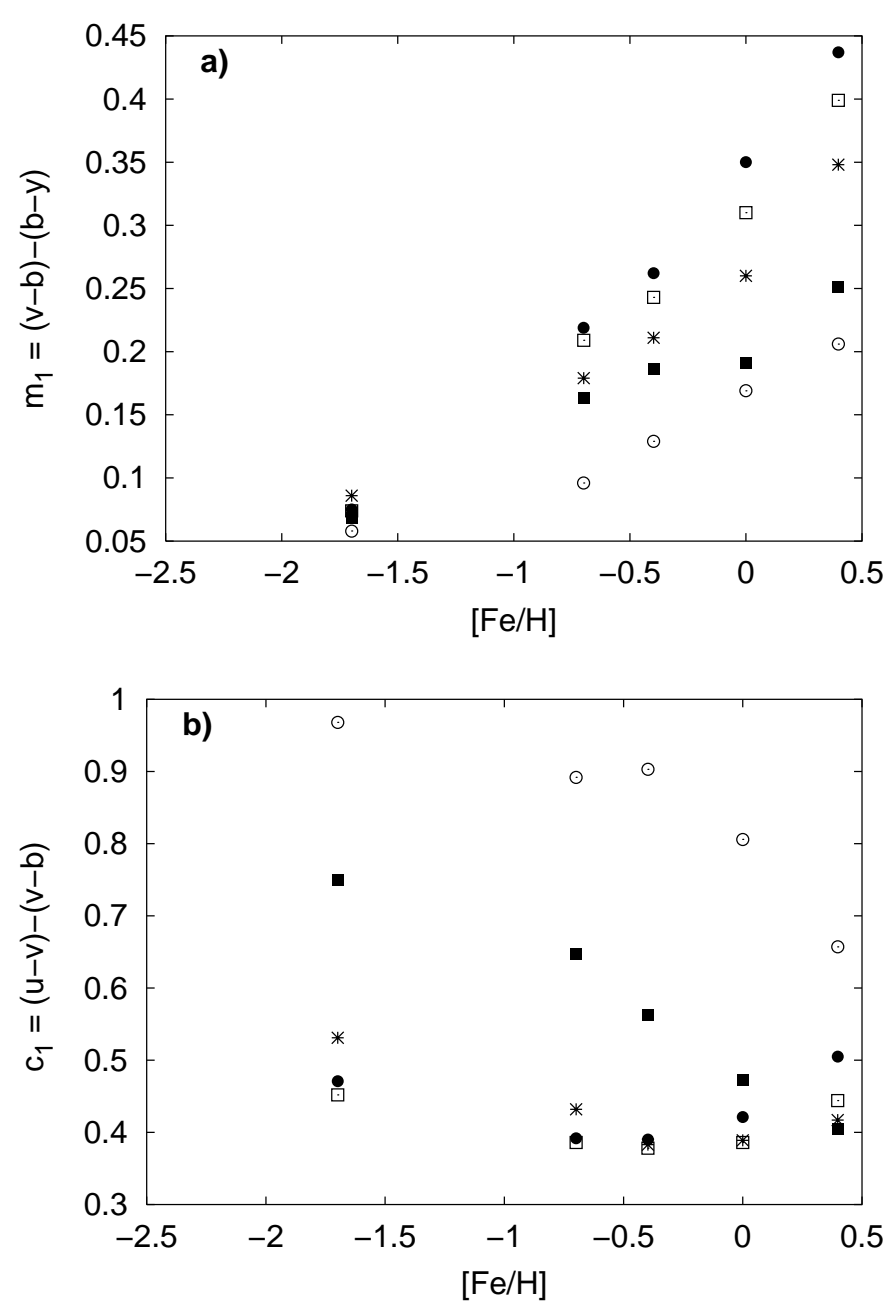

Fig. 10. a) Strömgren $m_{1}$ and b) $c_{1}$ color indices vs. metallicity $[\mathrm{Fe} / \mathrm{H}]$ for SSPs with Salpeter IMF at various ages. 0.5 Gyr (open circles), 1 Gyr (filled squares), 5 Gyr (stars), $10 \mathrm{Gyr}$ (open squares), and $14 \mathrm{Gyr}$ (filled circles).

While empirical calibrations like those obtained from Milky Way or M 31 GC analyses are only applicable for objects of comparatively old ages, our theoretical calibrations can also be used for intermediate age and young clusters, as long as emission lines from the active SF phase do no longer affect the broad band colors. From our extensive electronic tables, theoretical calibrations analogous to the ones depicted in Figs. 9a-c can be extracted for any color of our filter systems. They provide a useful tool for the interpretation of young star clusters forming in large numbers in interacting, merging, and starbursting gas-rich galaxies, for young and intermediate age GCs in old merger remnants and kinematically young elliptical and $\mathrm{S} 0$ galaxies. The extension to metallicities in the range $0.5 Z_{\odot} \ldots 2.5 Z_{\odot}$, that is only possible with theoretical calibrations, is of particular importance since spectroscopy of the first handful of bright young star clusters recently formed in spiral galaxy mergers has proven their relatively high metallicity - compared to Galactic GCs (e.g. Schweizer \& Seitzer 1993; Whitmore et al. 1999). Since these 2nd generation GCs are formed from gas pre-enriched in the interacting and/or
Table 2. Evolutionary and cosmological corrections $\epsilon_{\lambda}$ and $\kappa_{\lambda}$, respectively, as a function of redshift $z$ from $z=0$ to $z=5$ in the cosmology $H_{0}=65, \Omega_{0}=0.10$ for all Johnson bands for an SSP with solar metallicity and Salpeter IMF. Full table with all wavelengths bands and for all metallicities and two different cosmologies is given in the electronic version, at CDS, and on our web page.

\begin{tabular}{ccccccc}
\hline \hline$z$ & $\epsilon_{U}$ & $\kappa_{U}$ & $\epsilon_{B}$ & $\kappa_{B}$ & $\epsilon_{V}$ & $\ldots$ \\
\hline 0.03 & 1.0545 & 0.9175 & 1.0460 & 0.8815 & 1.0432 & \\
0.05 & 1.0870 & 0.8455 & 1.0726 & 0.7765 & 1.0658 & \\
0.08 & 1.1595 & 0.7653 & 1.1290 & 0.6863 & 1.1100 & \\
0.10 & 1.2110 & 0.6876 & 1.1570 & 0.6017 & 1.1200 & \\
0.13 & 1.2722 & 0.6121 & 1.1970 & 0.5275 & 1.1397 & \\
0.15 & 1.3439 & 0.5450 & 1.2510 & 0.4631 & 1.1738 & \\
0.17 & 1.4049 & 0.4767 & 1.2993 & 0.4103 & 1.2083 & \\
0.20 & 1.4646 & 0.4048 & 1.3455 & 0.3675 & 1.2442 & \\
0.23 & 1.5273 & 0.3400 & 1.3934 & 0.3296 & 1.2823 & \\
0.25 & 1.5904 & 0.2814 & 1.4434 & 0.2970 & 1.3226 & \\
$\vdots$ & & & & & & \\
\hline
\end{tabular}

starbursting galaxies, metallicities $\geq 0.5 Z_{\odot}$ were expected (Fritze - v. A. \& Gerhard 1994).

\section{Cosmological evolution}

In this section, we present evolutionary and cosmological corrections for direct use of our SSP results in combination with galaxy formation or cosmological structure formation scenarios. We chose two different cosmological models as characterised by $\left(H_{0}, \Omega_{0}, \Lambda_{0}\right)=(65,0.1,0.0)$ and $(75,0.1,0.9)$, respectively, and an assume an onset of SF at redshift $z_{\mathrm{f}}=5$. The manifold of cosmological models is constrained by the ages of Milky Way GCs (12-14 Gyr).

In case of galaxies, the luminosities of model galaxies at today's age of 12-14 Gyr are normalized to their observed average local luminosities, i.e. to the average local $\left\langle M_{B}\right\rangle$ of the respective galaxy type, and their evolutionary and cosmological corrections are then given in terms of magnitude differences. Such a normalization is not meaningful for SSPs if understood as galaxy constituents or subpopulations. Hence, as explained in Sect. 2.6, we chose to present their evolutionary and cosmological corrections $\left(\epsilon_{\lambda}, \kappa_{\lambda}\right)$ in terms of luminosity ratios in selected filter bands $\lambda$.

In Table 2 we present these corrections as a function of redshift $z$ for the Johnson $U \ldots K$ bands. They are given for the two cosmological models chosen above. Only the first few columns and lines of this table are shown for one cosmology in the printed version for orientation. Full tables are available in the electronic version, at CDS, and our web site.

The apparent magnitude $m_{\lambda}(z)$ in some filter $\lambda$ of an SSP of given mass and metallicity at redshift $z, m_{\lambda}(z)=$ $-2.5 \log L_{\lambda}(z, t(z))$ is obtained from the absolute luminosity $L_{\lambda}\left(0, t_{0}\right)$ of an SSP of given mass by today's age $t_{0}=$ $t(z=0)$ via

$m_{\lambda}(z)=-2.5 \log \left(L_{\lambda}\left(0, t_{0}\right) \cdot \epsilon_{\lambda}(z) \cdot \kappa_{\lambda}(z)\right)+B D M(z)$. 


\section{Conclusions}

A new set of evolutionary synthesis models are presented for single burst single metallicity stellar populations covering metallicities $0.02 \leq Z / Z_{\odot} \leq 2.5$ and ages $4 \times 10^{6} \mathrm{yr} \leq t \leq$ 16 Gyr. They are based on the most recent isochrones from the Padova group (Nov. 1999) that extend earlier models by the inclusion of the thermal pulsing AGB phase for stars in the mass range $2 M_{\odot} \leq m \leq 7 M_{\odot}$ in accordance with the fuel consumption theorem. We show that with respect to earlier models, inclusion of the TP-AGB phase leads to significant changes in the $(V-I)$ and $(V-K)$ colors of SSPs in the age range from $10^{8}$ to $\gtrsim 10^{9} \mathrm{yr}$, in particular at metallicities $Z \geq 0.5 Z_{\odot}$. The ages derived from observed $(V-I)$ colors of young star clusters in this age range decrease by a factor of $\sim 2$ when the TPAGB phase is included. Using model atmosphere spectra from Lejeune et al. (1997, 1998), we calculate the spectral evolution of single burst populations of various metallicities covering the wavelength range from $90 \AA$ through $160 \mu \mathrm{m}$. Expanding dust shells around AGB stars, however, are not accounted for by our models. Therefore, they should not be used for the interpretation of IR data (i.e. beyond $K$ ) of intermediate age star clusters. Age and metallicity effects of the spectral and photometric evolution of our SSP models are discussed in detail. Isochrone spectra are convolved with filter response functions to describe the time evolution of luminosities and colors in Johnson, Thuan \& Gunn, Koo, HST, Washington, and Strömgren filters.

Theoretical calibrations are provided for a number of colors in terms of metallicity and compared with empirical calibrations and data from GCs. It is found that the empirical calibrations are only suitable for the age and metallicity range of the clusters they are derived from. Our models do not have such restrictions and cover the whole metallicity - and age - range. They allow to study color - metallicity calibrations in their time evolution and up to metallicities $\geq Z_{\odot}$, both of which is important for the interpretation of young and intermediate age star cluster systems.

Model results are not only intended for use in the interpretation of star clusters over the full age range from few $10^{7}-1.4 \times 10^{10} \mathrm{yr}$ but also for combination with any kind of dynamical galaxy formation and/or evolution model that contains a star formation criterion, i.e. some prescription how to transform gas into stars. Moreover, the evolution of these single burst single metallicity stellar populations is readily folded with any kind of star formation - and eventually chemical enrichment - history to describe the evolutionary spectral synthesis of composite stellar populations like galaxies of any type with continuous or discontinuous SF.

For these latter purposes we also present the time evolution of mass-to-light ratios (including ejection rates for gas and metals) for two different IMFs as well as cosmological and evolutionary corrections for all the filters as a function of redshift $z$ for $0 \leq z \leq 5$ and two different cosmologies.

Extensive data files are provided in the electronic version, at CDS, and at http: //www . uni-sw.gwdg. de/ galev/.

Acknowledgements. We thank Doug Geisler for his interest and help with the calibration of the Washington system.

\section{References}

Barmby, P., Huchra, J. P., Brodie, J. P., et al. 2000, AJ, 119, 727

Bertelli, G., Bressan, A., Chiosi, C., Fagotto, F., \& Nasi, E. 1994, A\&AS, 106, 275

Bessel, M. S., \& Brett, J. M. 1988, PASP, 100, 1134

Bessel, M. S., Brett, J. M., Scholz, M., \& Wood, P. R. 1989, A\&AS, 77,1

Bessel, M. S., Brett, J. M., Scholz, M., \& Wood, P. R. 1991, A\&AS, 89,335

Bressan, A., Fagotto, F., Bertelli, G., \& Chiosi, C. 1993, A\&AS, 100, 647

Bressan, A., Granato, G. L., \& Silva, L. 1998, A\&A, 332, 135

Brodie, J. P., \& Huchra, J. P. 1990, ApJ, 362, 503

Bruzual, G. A., \& Charlot, S. 1993, ApJ, 405, 538

Chabrier, G., \& Baraffe, I. 1997, A\&A, 327, 1039

Charlot, S. 1996, in From Stars to Galaxies: The Impact of Stellar Physics on Galaxy Evolution, ed. C. Leitherer, U. Fritze - v. Alvensleben, \& J. Huchra, ASP Conf. Ser., 98, 275

Chiosi, C., Bertelli, G., \& Bressan, A. 1992, ARA\&A, 30, 305

Contardo, G., Steinmetz, M., \& Fritze - v. Alvensleben, U. 1999, ApJ, 507,497

Couture, J., Harris, W. E., \& Allwright, J. W. B. 1990, ApJS, 73, 671

Fagotto, F., Bressan, A., Bertelli, G., \& Chiosi, C. 1994, A\&AS, 104, 365

Fagotto, F., Bressan, A., Bertelli, G., \& Chiosi, C. 1994, A\&AS, 105,29

Fagotto, F., Bressan, A., Bertelli, G., \& Chiosi, C. 1994, A\&AS, 105,39

Fioc, M., \& Rocca-Volmerange, B. 1997, A\&A, 326, 950

Fluks, M. A., Plez, B., The, P. S., et al. 1994, A\&AS, 105, 311

Fritze - v. Alvensleben, U. 2000, in Massive Stellar Clusters, ed. A. Lançon, \& C. Boily, ASP Conf. Ser., 211, 3

Fritze - v. Alvensleben, U., \& Burkert, A. 1995, A\&A, 300, 58

Fritze - v. Alvensleben, U., \& Gerhard, O. E. 1994, A\&A, 285, 775

Geisler, D. 2000, priv. comm.

Girardi, L., \& Bertelli, G. 1998, MNRAS, 300, 533

Girardi, L., Bressan, A., Bertelli, G., \& Chiosi, C. 2000, A\&AS, 141, 371

Gray, R. O. 1998, AJ, 116, 482

Groenewegen, M. A. T., \& de Jong, T. 1993, A\&A, 267, 410

Gunn, J. E., \& Stryker, L. L. 1983, ApJS, 52, 121

Harris, H. C., \& Canterna, R. 1979, AJ, 84, 1750

Harris, W. E. 1996, AJ, 112, 1487

Koo, D. 1985, AJ, 90, 418

Kron, R. G. 1980, ApJS, 43, 305

Kurth, O. M., Fritze - v. Alvensleben, U., \& Fricke, K. J. 1999, A\&AS, 138, 19

Kurucz, R. L. 1992, IAU Symp., 149, 225

Lamla, E. 1982, in LB new Ser. VI/2B, 35

Lançon, A. 1999, IAU Symp., 191, 579

Lançon, A., \& Wood, P. R. 2000, A\&AS, 146, 217

Leitherer, C., Alloin, D., Fritze - v. Alvensleben, et al. 1996, PASP, 108, 996

Leitherer, C., Schaerer, D., Goldader, J. D., et al. 1999, ApJS, 123, 3

Lejeune, T., Cusinier, F., \& Buser, R. 1997, A\&AS, 125, 229

Lejeune, T., Cusinier, F., \& Buser, R. 1998, A\&AS, 130, 65

Rich, R. M., Shara, M. M., \& Zurek, D. 2001, AJ, 122, 842

Schweitzer, F., \& Seitzer, P. 1993, ApJ, 417, 29

Thuan, T. X., \& Gunn, J. E. 1976, PASP, 88, 543

Vallenari, A., Aparicio, A., Fagotto, F., \& Chiosi, C. 1994, A\&A, 284, 424

Vallenari, A., Richichi, A., Carraro, G., \& Girardi, L. 1999, A\&A, 349,825

Whitmore, B. C., Zhang, Q., Leitherer, C., et al. 1999, AJ, 118, 1551 